\title{
Assessing the potential of $S W V I$ (Soil Wetness Variation Index) for hydrological risk monitoring by means of satellite microwave observations
}

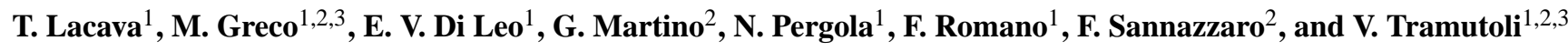 \\ ${ }^{1}$ Istituto di Metodologie per l'Analisi Ambientale - CNR, Tito Scalo (Potenza), Italy \\ ${ }^{2}$ Dip. di Ingegneria e Fisica dell' Ambiente, Università della Basilicata, Potenza, Italy \\ ${ }^{3}$ CIMA - Università della Basilicata, Università di Genova, Italy
}

Received: 14 November 2004 - Revised: 18 April 2005 - Accepted: 20 April 2005 - Published: 4 July 2005

\begin{abstract}
In the last years satellite remote sensing applications in hydrology have considerably progressed. A new multi-temporal satellite data-analysis approach has been recently suggested in order to estimate space-time changes of geophysical parameters possibly related to the increase of environmental and hydro-geological hazards. Such an approach has been already used both for flooded area mapping (using AVHRR data) and for soil wetness index estimation (using AMSU data).

In this work, a preliminary sensitivity analysis of the proposed Soil Wetness Variation Index $(S W V I)$ is made in the case of low intensity meteorological events by the comparison with hydrological (precipitation) data. This analysis, as a first step of a more complex work in progress, is targeted to a first evaluation of the reliability of the SWVI in describing soil response to precipitations of different duration and intensity.
\end{abstract}

\section{Introduction}

Soil moisture is the key state variable in hydrology: it is the switch that controls the proportion of rainfall that percolates, runs off or evaporates from the land. In particular, the knowledge of soil moisture and/or soil wetness is very important for flood prediction systems; runoff generation processes, in fact, are related to antecedent soil moisture conditions, especially regarding the water content at the very soil surface.

Precise in situ measurements of soil moisture are generally sparse and each value is only representative of a limited area. Furthermore, ground-based networks cannot guarantee global coverage at sustainable costs. Remote sensing products, if achievable with sufficient accuracy and reliability, would provide truly meaningful wide-area soil wetness/moisture data for hydrological studies over large conti-

Correspondence to: T. Lacava

(lacava@imaa.cnr.it) nental regions. Traditional satellite techniques for soil moisture monitoring exploit the advantages of microwave sensors data. At these frequencies, in fact, electro-magnetic radiation leaving the Earth's surface is practically not affected by clouds (in the absence of rain), allowing an all-weather and all-time (as the microwave signal is available both during the day and the night) capability of observing the Earth's surface conditions. Moreover, thanks to the large contrast between dielectric properties of water and dry soil, microwaves are highly sensitive to the water content in the soil (Eagleman and Lin, 1976; Jackson et al., 1981). On the other hand, the estimate of soil moisture by using microwave radiances is strongly influenced by surface roughness and vegetation cover, whose contributions to the measured signal can drastically reduce the sensitivity of the retrieval algorithms for soil moisture (Choudhury et al., 1979; Wang et al., 1983; Jackson and Schmugge, 1989; Schmugge et al., 2002).

An innovative Soil Wetness Variation Index $(S W V I)$ has been recently proposed (Lacava et al., 2004) using data acquired by the microwave radiometer AMSU (Advanced Microwave Sounding Unit) which flies aboard NOAA (National Oceanic and Atmospheric Administration) satellites. This index, based on a general approach for multi-temporal satellite data analysis (RAT - Robust AVHRR Techniques, Tramutoli 1998), seems to be able to overcome (or strongly reduce) the above mentioned limitations. The $S W V I$ has been already applied with good results, both in terms of reliability and sensitivity, in the observation of some extreme flooding events which occurred in the past in Europe (Lacava et al., 2005).

In this work, a preliminary $S W V I$ sensitivity analysis is proposed in order to study the relations, in the spacetime domain, between the proposed index and precipitations. It should be stressed that this work represents a preliminary stage of a more complex multiphase analysis whose subsequent development will be opened to the comparison with other ancillary information such as land cover maps, litho-geological maps, etc. This analysis has been performed for some precipitation events, documented by the 


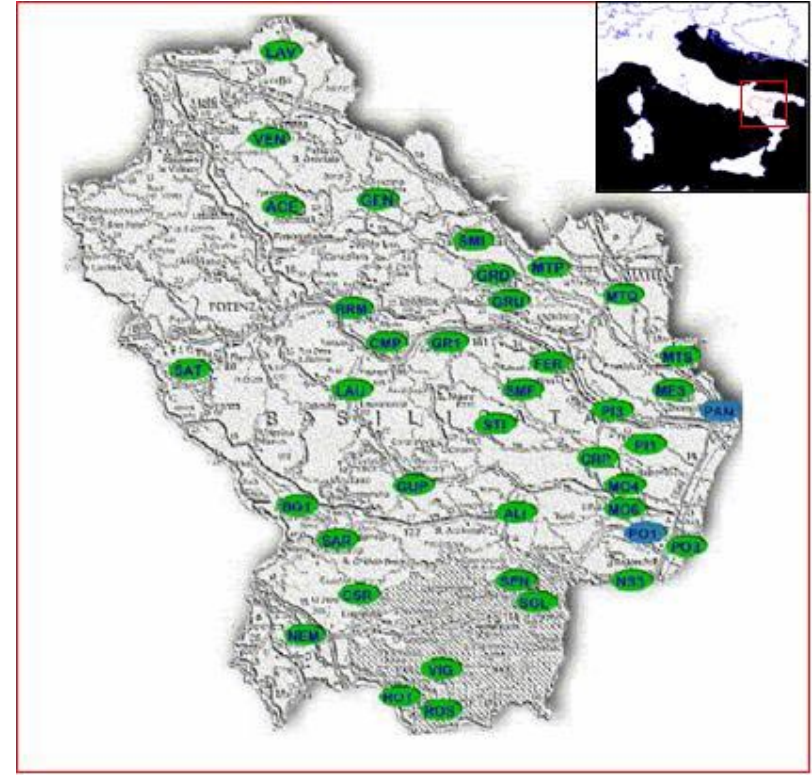

Fig. 1. The pilot area: Basilicata region in Southern Italy. In green and in blue are plotted the 40 measurement stations of Agenzia Lucana per lo Sviluppo e l'Innovazione in Agricoltura (ALSIA) measurement network (from http://www.alsia.it/servizi.htm).

micro-meteorological measurement network of the Agenzia Lucana per lo Sviluppo e l'Innovazione in Agricoltura (ALSIA), with different intensity and duration, which affected the Basilicata region located in the South Italy.

\section{The Soil Wetness Variation Index}

The RAT approach is a change-detection scheme which identifies signal anomalies in the space-time domain as deviations from a normal state that has been preliminarily identified (and usually given in terms of time average and standard deviation of the signal at sensor) on the basis of satellite observations collected during several years, under similar observational conditions, for each image pixel and in the same period of the year. Such an approach has already been successfully applied to several natural and environmental emergencies, such as volcanic eruptions (Pergola et al., 1998, 2001, 2004), forest fires (Cuomo et al., 2001) and earthquakes (Tramutoli et al., 2001; Di Bello et al., 2004; Filizzola et al., 2004). A detailed description of the theoretical background of RAT approach can be found in Tramutoli (1998).

RAT approach has been recently applied for soil wetness variation monitoring (Lacava et al., 2004, 2005). In these works, the proposed index was able to overcome all the above mentioned site effects, not only the ones due to surface roughness or vegetation cover, but also those due to the presence of permanent water bodies within the instantaneous field of view (IFOV) of the sensor. The same site observed under the same observational conditions, same month of year and same time of day, in fact, is expected to have the same roughness and quite similar vegetation cover, so that the possible residual emissivity variations observed can be mainly attributed to moisture variations in the soil (Lacava et al., 2004).

The Soil Wetness Variation Index $(S W V I)$ has been defined as:

$S W V I(x, y, t)=\frac{S W I(x, y, t)-\mu_{S W I}(x, y)}{\sigma_{S W I}(x, y)}$

where: $\operatorname{SWI}(x, y, t)$ is the Soil Wetness Index proposed by Grody $(2002)^{1}$ that identifies as wet all the pixels in an AMSU image which have values of the difference $\left(S W I=T_{89 \mathrm{GHz}}-T_{23 \mathrm{GHz}}\right.$ ) between the radiance (expressed in Brightness Temperature) measured in the AMSU channels 15 (at $89 \mathrm{GHz}$ ) and 1 (at $23 \mathrm{GHz}$ ), respectively, higher than $5 \operatorname{Kelvin} ; \mu_{S W I}(x, y)$ is its time average and $\sigma_{S W I}(x, y)$ is the standard deviation describing its natural fluctuations, both computed on a selected multi-annual AMSU imagery data-set, composed only of AMSU data collected during the same month of the year and acquired at around the same hour of the day, according to the RAT prescriptions (Tramutoli 1998). The $S W V I(x, y, t)$ gives, for each pixel $(x, y)$ and time $t$ of observation, the actual $S W I$ excess compared to its unperturbed conditions, weighted by the normal variability, historically observed under similar observational conditions. The site effects due to surface roughness or vegetation cover as well as to the presence of permanent water bodies within the IFOV, are in this way strongly reduced, as the $\operatorname{SWVI}(x, y, t)$ index is solely sensitive to $S W I$ variations (for each place mainly depending on soil moisture) and not to its absolute value (strongly depending instead also on surface roughness and vegetation cover). We expect, then, that higher values of $S W V I(x, y, t)$ will be associated to the relative increase of soil wetness at each specific location. More specifically, positive $S W V I$ values would indicate soil wetter than "normal" conditions. In order to reduce possible false alarms, we have chosen to classify as moderately wet (respect to the unperturbed conditions) all those pixels with $S W V I$ values between 2 and 3 . Pixels with $S W V I$ values greater than 3 and lower than 4 suggest (anomalous) wet conditions, while those with $S W V I$ values higher than 4 should describe extremely wet conditions. However, it should be noted that, being $S W V I$ a qualitative index describing relative, rather than absolute conditions, higher positive $S W V I$ not necessarily identify soils close to the saturation state.

\section{The sensitivity analysis}

In this work a preliminary sensitivity analysis of the proposed $S W V I$ is presented. This analysis, performed by the comparison of the proposed satellite indicator with hydrological data, will allow us to give a first assessment of the reliability of $S W V I$ in describing soil response to no-extreme precipitation events with different duration and intensity.

\footnotetext{
${ }^{1}$ Grody, N. C.: An AMSU Soil Wetness Index, (private communication), 2002.
} 

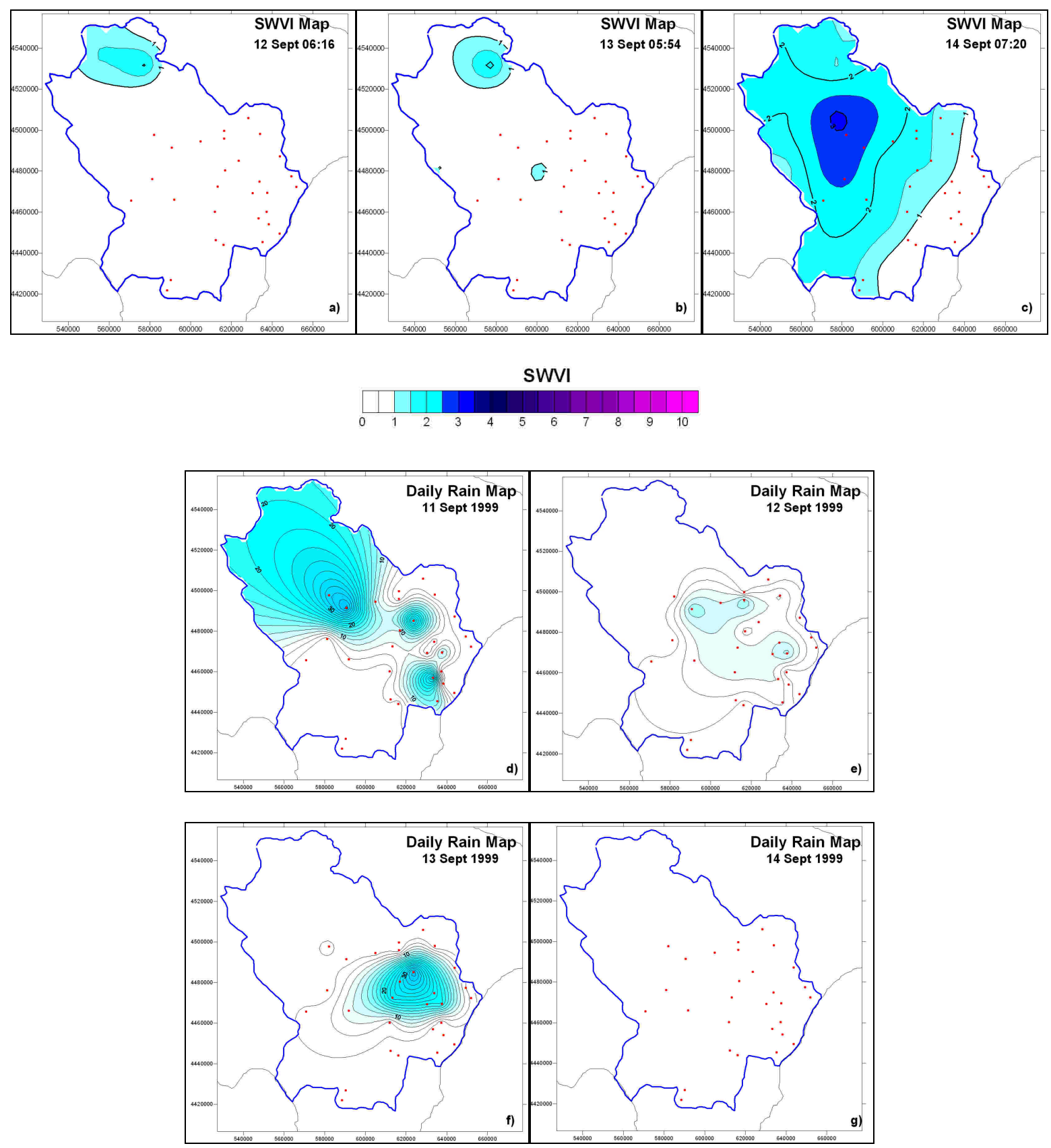

Daily rain [mm]

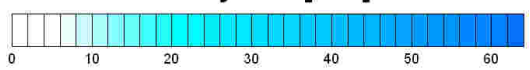

Fig. 2. Sensitivity analysis for the September 1999 event: (a), (b), (c) SWVI maps respectively for 12th, 13th and 14th September; (d), (e), (f), (g) regional cumulative rainfall map for 11th, 12th, 13th, 14th September respectively. Red dots identify ALSIA network measurement stations. Colour bars show the whole range of fluctuation for each field. All the maps are in the same geographic projection (UTM-WGS84). Note the quite good spatial-temporal correlation between the measured rainfall and the $S W V I$. Highest $S W V I$ values are in fact present in the image of September 14th after the precipitation fallen in the previous days.

The pilot area, represented in Fig. 1, is the Basilicata region in Southern Italy and the hydrological data, provided by the ALSIA rain gauges network which, through 40 measurement stations working during 2004, covers the region at whole. In this work we use only the data coming from 29 measurement stations which, working since 1998, have a more complete temporal overlapping with AMSU historical data-set. AMSU data, in fact, are available since 1998, 
when NOAA 15 has been launched. NOAA 15 ensures two passes per day, one around 5:00 GMT and another one around 17:00 GMT (12 h later). In order to generate references fields, we considered two different data sets, one including all the imagery acquired during the morning passes, the other one using the afternoon passes. The images acquired during the months of events occurrences have been analysed for the whole period of AMSU data availability (since 1998 up to now) processing, in average, about 240 passes per each event (120 for each data set).

The main idea, in order to perform the sensitivity analysis, is to relate the computed value of SWVI to the observed rainfall for some precipitation events which have been preliminarily selected (the ones occurred in September 1999, April 2001 and November 2002). As a first step of the full analysis (in progress), only a qualitative correlation has been carried out, neglecting, at this stage, other possible input variables (i.e. land cover maps, digital terrain models, etc.) and/or tuning parameters (i.e. variable temporal lag, different cumulating periods, etc.) which will be considered in the next phases.

Preliminary comparisons have been performed analysing the correlation between the daily $(24 \mathrm{~h})$ cumulative rainfall data (used as input for the kriging technique applied to generate the regional daily rainfall map) and the corresponding spatial distribution of the SWVI for each available AMSU image acquired the same day. It should be stressed (and taken into account analysing the results) that the $S W V I$ is an instant observation, referred to a specific acquisition time of the day, whereas the rainfall map is considered here as a "daily" observation, obtained cumulating all the available data recorded during the same day.

\subsection{Results}

Results obtained for the events occurred during 11-14 September 1999, 12-16 April 2001 and 18-19 November 2002 and they are shown in the next figures and discussed in the following.

Figure 2 shows both the maps of $S W V I$ and the regional cumulative rainfall maps referring to September 1999 event. The analysis of the figure highlights a good spatial and temporal correlation between the rainfall and the proposed index. The northern part of the region, in fact, is characterized by anomalous values of $S W V I$ which reach their maximum values, both in terms of intensity and spatial distribution, in the image of 14 September, as a direct consequence of the precipitation that affected the area in the previous days (Figs. $2 \mathrm{~d}-\mathrm{g}$ ). This is a first indication of the potential of the proposed index because, although the clearly non-extreme nature of the considered precipitation event (both in terms of duration and intensity) the $S W V I$ was sensitive enough to record a significant variation (higher than 3 sigma over the unperturbed values expected for those places in this season) in soil wetness conditions.

In Fig. 3 the results obtained by the analysis of April 2001 event are shown. Note how, after the precipitation which affected the Basilicata region during 11-12 April, high values of SWVI appear (Fig. 3a). Rainfall interested the whole area in the following days reaching their top on 14 April. In the first AMSU image available after this date (on 16 April), pixels with anomalous values of $S W V I$ are shown again, appearing in quite a good spatial correlation with the actual precipitations. Moreover, it should be stressed, as an additional result that, although the geographical area investigated was greater than the region of interest, no false positive $S W V I$ (false alarms) have been identified in any of the considered AMSU scenes.

Figure 4 reports the results for the analysis of the November 2002 event. In detail, in the figure is shown the response of $S W V I$ for 17 November, just before the storm occurred the day after (18 November), outlining an almost "dry" condition of the area confirmed also by the absence of significant rainfall (Fig. 4d). Looking at Fig. 4b, which refers to the AMSU scene of 18 November, is clearly detected the presence of a raining cloud system located over Basilicata region (identified by a screening test proposed by Grody et al. (2000) using AMSU data), confirmed by the investigation of rain-gauge network data as well (Fig. 4e reveals a significant storm occurred on 18 November) In the SWVI map of 19 November (Fig. 4c) a lightly perturbed condition for $S W V I$ within the investigated area is shown, confirming once more the timely sensitivity of the proposed index to the preceding rainfall.

In conclusion, the preliminary results obtained in this work, although they need further assessments over extended sets of events, seem to confirm that also no-extreme precipitation events, confined in time and space, like those above described may produce a significant response of the $S W V I$ which shows, then, quite a good sensitivity to such events as well. Moreover, this work represents a further confirmation of the reliability of the proposed index which, in all the three considered study cases, resulted completely false alarms-proof.

\section{Conclusions}

In this paper, preliminary results of a sensitivity analysis of a new satellite surface wetness index (the $S W V I$ ) are presented. The $S W V I$, generated by a multi-temporal analysis of AMSU data, has been already applied with encouraging results to the observations of some flooding events occurred in the past in Europe. The qualitative sensitivity analysis, performed in this work by comparison with rain gauge data obtained by a dense ground network located in Basilicata Region (Southern Italy), allow us to give a first assessment of the capabilities of the $S W V I$ in describing soil response to precipitations of different duration and intensity.

The first results achieved in this work, obtained by means of a correlation analysis with precipitation data during some minor raining events occurred in Basilicata region, encourage to continue research in this field, suggesting the possibility to employ the $S W V I$ as a further parameter to be used in the prediction chain devoted to the mitigation of the hydrological risk. 

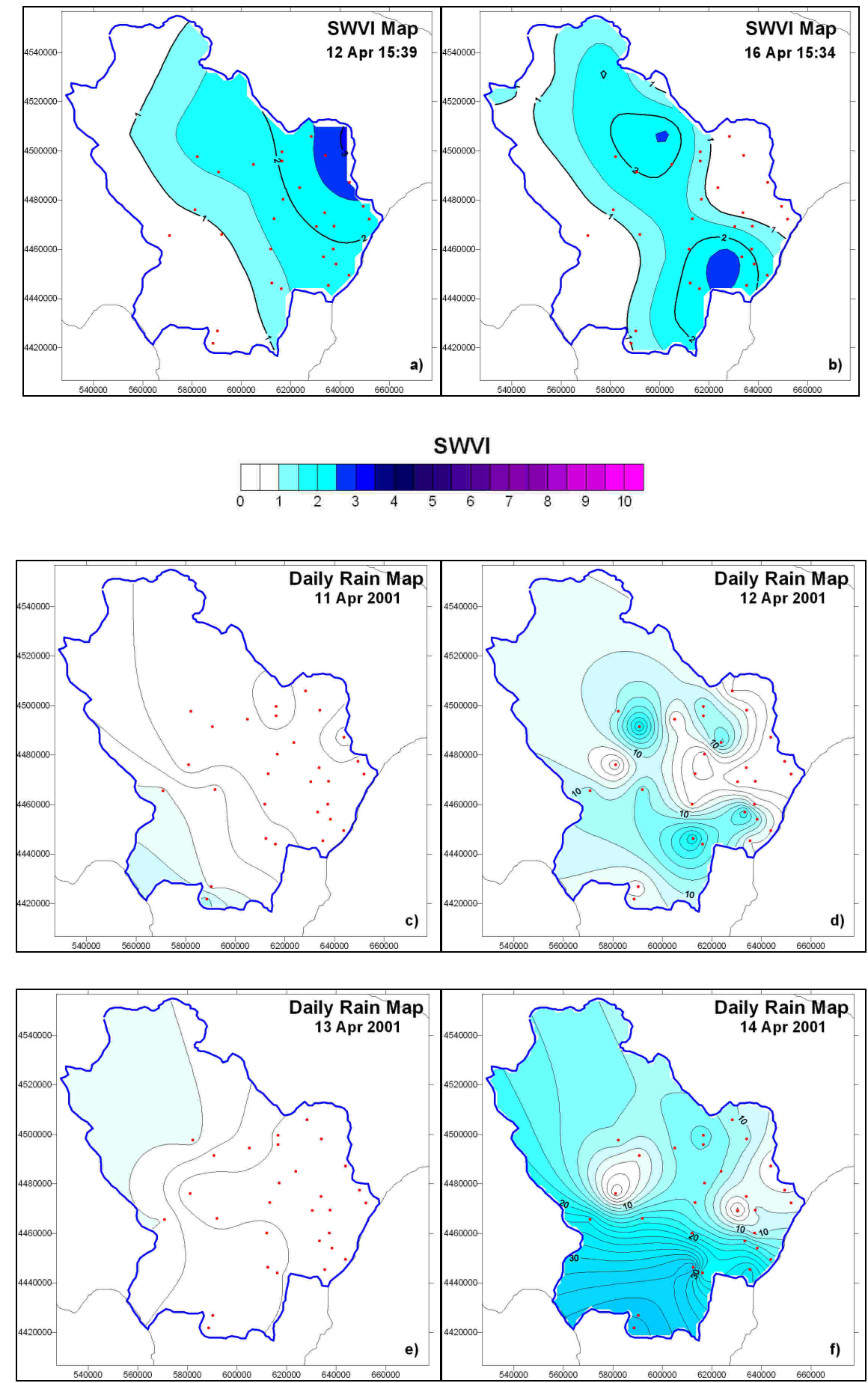

\section{Daily rain [mm]}

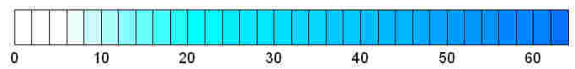

Fig. 3. Sensitivity analysis for the April 2001 event: (a), (b) SWVI maps for 12th, 16th April; (c), (d), (e), (f) regional cumulative rainfall map respectively for 11th, 12th, 13th, 14th April. Red dots identify ALSIA network measurement stations. Colour bars show the whole range of fluctuation for each field. All the maps are in the same geographic projection (UTM-WGS84). The whole region was affected by rainfall mainly on 12 and 14 April 2001, consequently anomalous SWVI values appear in the images of 12th and 16th April. 

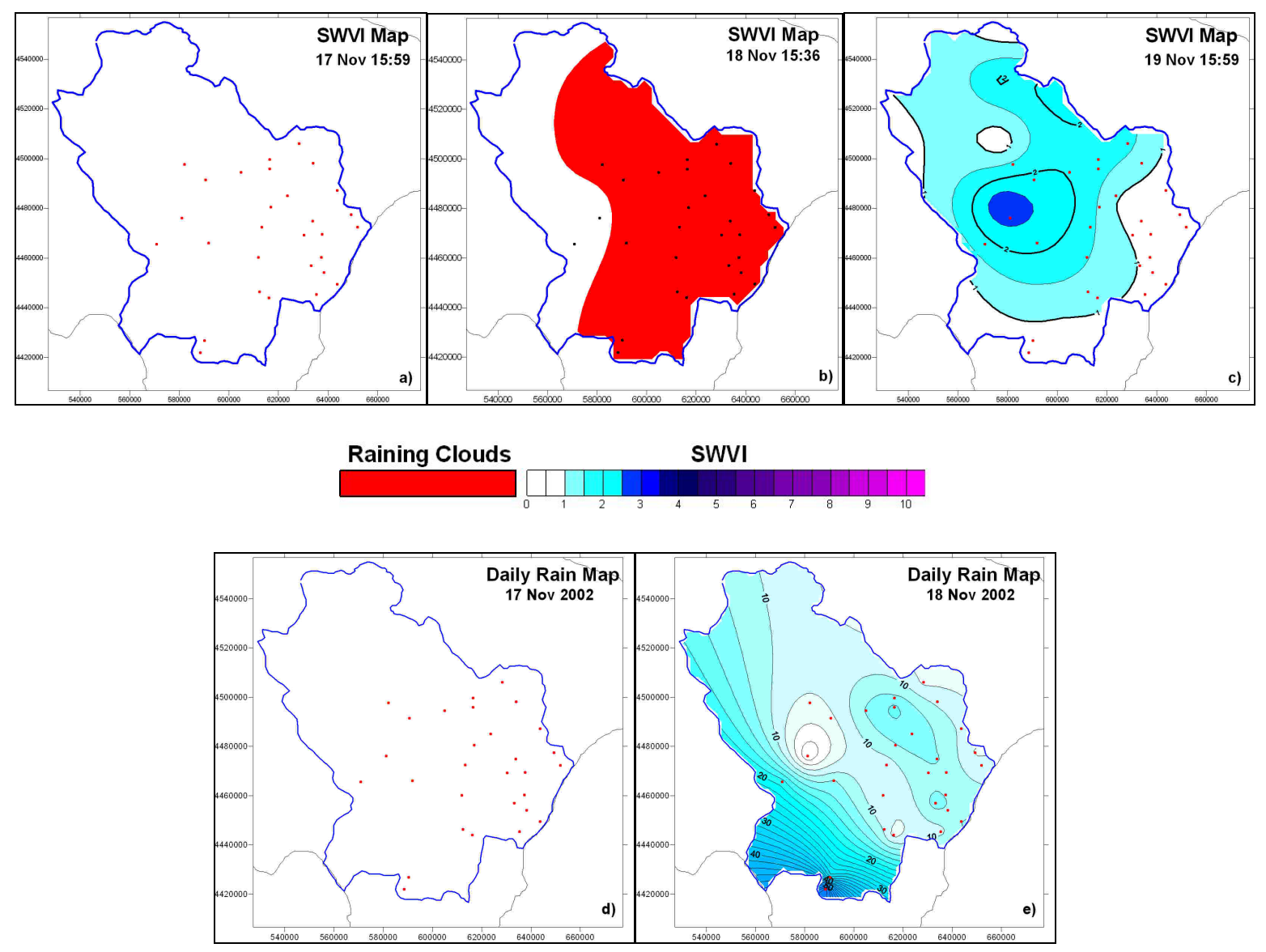

Daily rain $[\mathrm{mm}]$

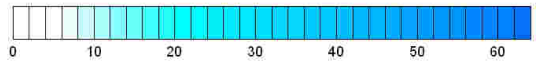

Fig. 4. Sensitivity analysis for the November 2002 event: (a), (b), (c) $S W V I$ maps for 17th, 18th and 19th November; (d), (e) regional cumulative rainfall map for 17th and 18th November respectively. Red dots identify ALSIA network measurement stations. Colour bars show the whole range of fluctuation for each field. All the maps are in the same geographic projection (UTM-WGS84). In Fig. 4b, the soil is masked by the raining clouds affecting the region (they have been identify by a screening test proposed by Grody et al. (2000), see text). Note as after the intense precipitation fallen during 18th November, intense SWVI values consequently appear (on the 19th November map).

In the next future, a switch from a qualitative sensitivity analysis to a quantitative study, already planned, will give us more reliable and detailed indications. This will be achieved by means of a point-to-point 2-D correlation analysis, carried out giving more importance to other local input data, like land cover maps, litho-geological data, digital terrain model. Moreover, additional tuning parameters will be taken into account, as for instance variable temporal lags and different cumulating periods, in order to better assess the actual potential of the proposed indicator for a reliable, timely and efficient monitoring of soil wetness which, although its crucial role, still remains one of the main missing information.

Edited by: L. Ferraris

Reviewed by: anonymous referees

\section{References}

Agenzia Lucana di Sviluppo e di Innovazione in Agricoltura, http: //www.alsia.it/, 2000.

Choudhury, B. J., Schmugge, T. J., Chang, A., and Newton, R. W.: Effects of Surface Roughness on the Microwave Emission from Soil, J. Geophys. Res., 84 (C9), 5699-5706, 1979.

Cuomo, V., Lasaponara, R., and Tramutoli, V.: Evaluation of a new satellite-based method for forest fire detection, Int. J. Rem. Sens., 22 (9), 1799-1826, 2001.

Di Bello, G., Filizzola, C., Lacava, T., Marchese, F., Pergola, N., Pietrapertosa, C., Piscitelli, S., Scaffidi, I., and Tramutoli, V.: Robust Satellite Techniques for Volcanic and Seismic Hazards, Annals of Geophysics, 47 (1), 49-64, 2004.

Eagleman, J. R. and Lin, W. C.: Remote Sensing of Soil Moisture by a 21-cm Passive Radiometer, J. Geophys. Res., 81, 36603666, 1976.

Filizzola, C., Pergola, N., Pietrapertosa, C., and Tramutoli, V.: Robust satellite techniques for seismically active areas monitoring: 
a sensitivity analysis on September 7th 1999 Athens's earthquake, Special issue on Seismo Electromagnetics and Related Phenomena, Phys. Chem. Earth, 29 (4-9), 517-527, 2004.

Grody, N. C., Weng, F., and Ferraro, R. R.: Application of AMSU for hydrological parameters, in: Microwave radiomemetry and remote sensing of the earth's surface and atmosphere, edited by: Pampaloni, P. and Paloscia, S., VSP, The Netherlands, 339-352, 2000.

Jackson, T. J., Schmugge, T. J., Nicks, A. D., Coleman, G. A., and Engman, E. T.: Soil Moisture Updating and Microwave Remote Sensing for Hydrological Simulation, Hydrological Sciences Bulletin, 26 (3), 305-319, 1981.

Jackson, T. J. and Schmugge, T. J.: Passive Microwave Remote Sensing System for Soil Moisture: Some Supporting Research, IEEE Trans. Geosci. Rem. Sens., 27, 225-235, 1989.

Lacava, T., Di Leo, E. V., Pergola, N., Romano, F., Sannazzaro, F., and Tramutoli, V.: Analysis Of Multi-Temporal Satellite Records For Extreme Flooding Events Monitoring, Proceedings of the 5th EGS Plinius Conference held at Ajaccio, Corsica, France, October 2003, edited by: Testud, J., Mugnai, A., and Santucci, J. F., 231-237, 2004.

Lacava, T., Cuomo, V., Di Leo, E. V., Pergola, N., Romano, F., and Tramutoli, V.: Improving soil wetness variations monitoring from passive microwave satellite data: the case of April 2000 Hungary flood, Rem. Sens. Environ., accepted, 2005.
Pergola, N., Pietrapertosa, C., and Tramutoli, V.: Satellite Remote Sensing of Volcanic Aerosol: a new, AVHRR-based, approach, Satellite Remote Sensing of Clouds and Atmosphere III, edited by: Russel, J. E., Proceedings of SPIE, 3495, 188-197, 1998.

Pergola, N., Pietrapertosa, C., Lacava, T., and Tramutoli, V.: Robust Satellite Techniques for Volcanic Eruptions Monitoring, Annals of Geophysics, 44 (2), 167-177, 2001.

Pergola, N., Lacava, T., Marchese, F., Scaffidi, I, and Tramutoli, V.: Improving volcanic ash cloud detection by a robust satellite technique, Rem. Sens. Env., 90, 1-22, 2004.

Schmugge, T. J., Kustas, W. P, Ritchie, J. C., Jackson, T. J., and Rango, A: Remote Sensing in hydrology, Adv. Water Res., 25, 1367-1385, 2002.

Tramutoli, V.: Robust AVHRR Techniques (RAT) for Environmental Monitoring theory and applications, in: Earth Surface Remote Sensing II, edited by: Cecchi, G. and Zilioli, E., SPIE 3496, 101113, 1998.

Tramutoli, V., Di Bello, G., Pergola, N., and Piscitelli, S.: Robust Satellite Techniques for Remote Sensing of Seismically Active Areas, Annals of Geophysics, 44 (2), 295-312, 2001.

Wang, J. R., O’Neill, P. E., Jackson, T. J., and Engman, E. T.: Multifrequency Measurements of the effects of Soil Moisture, Soil Texture and Surface Roughness, IEEE Trans. Geosci. Rem. Sens., GE-21(1), 44-51, 1983. 